\title{
Transplantation of Lymphocytes Co-Cultured with Human Cord Blood-Derived Multipotent Stem Cells Attenuates Inflammasome Activity in Ischemic Stroke
}

This article was published in the following Dove Press journal:

Clinical Interventions in Aging

\section{Yanxin Zhao* \\ Tianrui Zhu* \\ Heng $\mathrm{Li}$ \\ Jing Zhao \\ Xiaohong Li}

Department of Neurology, Jinan Central Hospital Affiliated to Shandong University, Jinan, Shandong 2500I3,

People's Republic of China

*These authors contributed equally to this work
Correspondence: Xiaohong Li

Department of Neurology, Jinan Central Hospital Affiliated to Shandong University,

No. 105 Jiefang Road, Jinan, Shandong

2500 I3, People's Republic of China

Tel +86-053I-68623309

Fax +86-053I-85695219

Email xiaohong-li@sdu.edu.cn
Background: Manipulating the immune inflammatory response after cerebral ischemia has been a novel therapeutic strategy for ischemic stroke. This study attempted to investigate the effects of the transplantation of lymphocytes co-cultured with human cord blood-derived multipotent stem cells (HCB-SCs) on the inflammatory response in transient middle cerebral occlusion (tMCAO) rats.

Methods: The tMCAO rats were subjected to the transplantation of lymphocytes co-cultured with HCB-SCs through tail vein injection. Infarct size and neurological deficits were measured at $48 \mathrm{hrs}$ after stroke. Neurological deficits were assessed using Bederson's scoring system and tape removal test. Blood $\mathrm{T}$ cell flow cytometry was performed to measure the differentiation of regulatory $\mathrm{T}$ cells (Tregs). Western blot was used to detect the protein levels of inflammationrelated molecules, apoptosis-related molecule, and signaling molecules in ischemic brain. TUNEL staining was performed to analyze cell apoptosis in ischemic cerebral cortex.

Results: The transplantation of lymphocytes co-cultured with HCB-SCs significantly improved the neurological defects, reduced ischemic brain damage, and increased the proportion of peripheral $\mathrm{CD}^{+} \mathrm{CD} 25^{+} \mathrm{Foxp}^{+}$Tregs. Meanwhile, the transplantation of co-cultured cells decreased the expression of NLRP3 inflammasome and associated factors, such as caspase-1 and IL- $1 \beta$, and inhibited the activation of NF- $\kappa \mathrm{B}$, ERK and caspase- 3 in ischemic brain. The cocultured cells significantly decreased the number of tMCAO-induced cell apoptosis.

Conclusion: Lymphocytes co-cultured with HCB-SCs exhibit a neuroprotective effect after ischemic stroke by promoting Tregs differentiation and suppressing NLRP3 inflammasome activation and neuron apoptosis, and might be a promising therapeutic strategy for ischemic stroke. Keywords: ischemic stroke, inflammation, cord blood-derived multipotent stem cells, regulatory T-cells, inflammasomes

\section{Introduction}

Stroke is a leading cause of death and permanent disability worldwide. ${ }^{1}$ The estimated global lifetime risk of stroke for those aged 25 years or older has increased from $22.8 \%$ in 1990 to $24.9 \%$ in 2016, and China has the highest incidence worldwide, with a risk of $39.3 \% .{ }^{1}$ Due to the growing and ageing population and increased prevalence of risk factors, there are excessive stroke burden globally. ${ }^{2-4}$ Currently, intravenous thrombolysis and thrombectomy have shown clear efficacy for ischemic stroke patients, and advances in recanalization 
therapies have extended the time window for the intervention of ischemic stroke. ${ }^{5}$ Whereas, a low number of stroke patients are eligible for recanalization treatment, and cerebral injury caused by ischemia-reperfusion is still a burning problem. Together, currently available therapies are insufficient for cerebral injury after stroke, strongly suggesting novel treatment options.

Inflammatory events are currently known to play critical roles in the progression of cerebral injury after stroke. ${ }^{6}$ Growing evidence suggests the manipulation of lymphocytes in ischemic stroke as a potential therapeutic strategy for the management of stroke. ${ }^{7-9}$ Inflammatory lymphocytes contribute to neuronal death and poor outcomes after stroke, and blocking the brain invasion of inflammatory lymphocytes is neuroprotective after ischemia-reperfusion. ${ }^{10,11}$ While, regulatory $\mathrm{T}$ lymphocytes (Tregs) have been characterized as disease-limiting protective cells, ${ }^{12-15}$ and play a key part in controlling immune responses in ischemic stroke. ${ }^{16}$ Therefore, increasing the proportion of Tregs is conducive in the immunomodulatory therapy for stroke.

Stem cell therapy is a promising and attractive alternative for repairing stroke-induced neurological damage. ${ }^{17}$ Various stem cells derived from different sources have been employed as donor cells for transplantation therapy in stroke. ${ }^{18-21}$ During the past years, human cord blood-derived multipotent stem cells (HCB-SCs) have emerged as a highly promising source for cell therapy in a variety of diseases. ${ }^{22-26}$ Experimental and preclinical studies have reported promising data of HCB-MSCs in stroke therapy, including improved neurobehavioral functions, reduced infarct volume, and prolonged survival. ${ }^{18,19,23,27}$ Moreover, HCB-SCs present unique superiorities, such as greater availability, less immunogenicity, and lower risk of mediating viral transmission, compared to other sources for stem cell therapy. ${ }^{26,28}$ Nevertheless, a potential limitation of stem cell transplantation is the predisposition of eliciting a graft-induced immunologic response from the host. Current suboptimal countermeasure mainly focuses on the immunosuppressive treatment for the host in experimental studies. ${ }^{27,29}$ It is critical to manipulate the graft to achieve beneficial improvements simultaneously with an immune homeostasis.

Increasing evidence has shown that HCB-SCs can modulate immunologic responses by altering Tregs. ${ }^{30,31}$ Our previous study discovered that HCB-SCs could increase the proportion of Tregs in peripheral lymphocytes, and lymphocytes co-cultured with HCB-SCs could improve pathological impairment of APP/PS1 mice via an immunomodulatory effect. ${ }^{25}$ Given the potency of HCB$\mathrm{SCs}$ and Tregs under cerebral ischemic condition, rat spleen lymphocytes were co-cultured with HCB-SCs in this study, and then co-cultured lymphocytes were intravenously transplanted into the rat stroke model of middle cerebral artery occlusion and reperfusion (MCAO). Meanwhile, we observed the modulating effect of HCB$\mathrm{SCs}$ on lymphocytes, the neuroprotective effect of cocultured lymphocytes as well as the underlying mechanism in modulating immune responses in ischemic stroke.

\section{Materials and Methods}

\section{Experimental Animals}

Male Wistar rats (weighing 250 300 g) were obtained from Beijing HFK Bioscience Co., LTD (Beijing, China). All rats were kept under a 12:12 hrs light/dark cycle at a constant temperature of $22^{\circ} \mathrm{C}$ and were free access to food and water. This study was approved by the Ethical Committee of School of Medicine, Shandong University. All animal experiments were performed in accordance with the Guide for the Care and Use of Laboratory Animals of Shandong University. The rats were randomly divided into 3 groups as follows: sham-operated group (Sham, $\mathrm{n}=10$ ), transient middle cerebral artery occlusion and reperfusion group ( $\mathrm{tMCAO}, \mathrm{n}=12$ ), and tMCAO treated with the transplantation of lymphocytes co-cultured with HCB-SCs (tMCAO+LT, n=12).

\section{tMCAO Model}

The rats were anesthetized with $10 \%$ chloral hydrate $(3 \mathrm{~mL} / \mathrm{kg}$ ) by intraperitoneal injection. The left common carotid artery was identified and isolated through a ventral midline cervical incision. A nylon filament $(0.28 \mathrm{~mm}$ in diameter) with a rounded tip was introduced from the common carotid artery lumen into the internal carotid artery to block the origin of the left middle cerebral artery (MCA). The left MCA was occluded with the filament for $90 \mathrm{~min}$, and the filament was withdrawn to allow MCA reperfusion. In the sham-operated group, the left neck incision was made to expose the arteries, but the nylon filament was not inserted into internal carotid artery.

\section{Cell Co-Culture and Transplantation}

The preparation and co-culture of HCB-SCs and spleen lymphocytes were conducted according to our previous report. ${ }^{25}$ Human cord blood collection for research was approved by the institutional review board of Shandong 
University and Jinan Central Hospital. Cord blood samples were collected from the placenta of healthy donors in Department of Obstetrics, Jinan Central Hospital with donors' written informed consent. The rat mononuclear lymphocytes were co-cultured with $\mathrm{HCB}-\mathrm{SCs}$ at a ratio of 10:1 in vitro for 3 days.

The rats were anesthetized with isoflurane $\left(2 \sim 5 \%\right.$ in $\mathrm{O}_{2}$ delivered at $2 \mathrm{~L} / \mathrm{min}$ ). Cells were adjusted to a concentration of $2 \times 10^{7}$ cells $/ \mathrm{mL}$. The rats received administration of lymphocytes co-cultured with HCB-SCs through tail vein injection at a final dose of $1 \times 10^{7}$ cells $/ 500 \mu \mathrm{L}$ saline once at $2 \mathrm{hrs}$ and $24 \mathrm{hrs}$ after reperfusion. The rats in the tMCAO group received saline injection without lymphocytes. An immunosuppressant was not given to the rats.

\section{Neurological Deficits Measurement Bederson's Scale Scores}

The behavioral assay was scored using a modified Bederson's test. ${ }^{32}$ At 48 hrs after reperfusion, the rats were examined by suspending $20 \sim 30 \mathrm{~cm}$ above the testing platform. The neurological deficits were scored according to the following criteria: 0 , rat extends both forelimbs straight; 1 , rat keeps one forelimb to the breast and extends the other forelimb straight; 2, rat shows decreased resistance to a lateral push without circling; 3 , rat shows decreased resistance to a lateral push with circling; and 4, rat loses spontaneous walking and consciousness. Rats scoring 0 or 4 were not used in the evaluation of this experiment.

\section{Tape Removal Test}

The tape removal test was performed to assess forepaw sensorimotor deficits at $48 \mathrm{hrs}$ after tMCAO. ${ }^{33}$ Rats were placed into a box $(60 \mathrm{~cm} \times 50 \mathrm{~cm})$. Two adhesive tapes with equal length $\left(113 \mathrm{~mm}^{2}\right)$ were quickly attached at the distal radial region of 2 forelimbs; then, the rats were placed into the box. The time that it took for an animal to remove the tapes from each forelimb was recorded. A rat was tested three times, and the median was taken for statistics. If an animal could not remove the tapes within $180 \mathrm{~s}$, then time was noted as $180 \mathrm{~s}$. Rats were trained before surgery for 3 days.

\section{Measurement of Infarct Volume}

After $48 \mathrm{hrs}$ of reperfusion, the rats were deeply anesthetized with $10 \%$ chloral hydrate. The brains were removed and sliced into $2.0 \mathrm{~mm}$ thick coronal sections. The brain slices were immediately incubated in $0.9 \%$ saline with $2 \%$ triphenyl tetrazolium chloride (TTC, Solarbio, China) at $37^{\circ} \mathrm{C}$ for $30 \mathrm{~min}$. Then the slices were transferred into $4 \%$ paraformaldehyde solution and fixed for $12 \mathrm{hrs}$. The photographs were taken by the scanner. Areas of the infarct regions were analyzed in Image-Pro Plus 6.0. The infarct areas of each section were the average of the sum of two sides. The volume of infarction for each animal was calculated by taking the product of the average slice thickness $(2 \mathrm{~mm})$ and summing the infarct areas in all brain slices. The results were represented as the percentage of the total volume.

\section{Flow Cytometry}

Peripheral blood was harvested in tubes with heparin sodium through angular veins. After centrifugation at $4^{\circ}$ C (1000 rpm, $30 \mathrm{~min})$, the supernatant plasma was collected and stored at $-80^{\circ} \mathrm{C}$ for further detection. For flow cytometry, the resuspended lymphocytes were isolated by rat lymphocyte separation medium (Solarbio, China) according to the manual. After washing, the cells were incubated with antibodies at $4{ }^{\circ} \mathrm{C}$ for $30 \mathrm{~min}$ as recommended by the manufacturer. The antibodies included antirat FITC-conjugated CD4, anti-rat AF647-conjugated CD25 and anti-rat PE-conjugated Foxp3. These antibodies were purchased from BioLegend Inc. Cell fixation and staining methods referred to the manufacturer's instruction. All samples were analyzed by a flow cytometer (Beckman CytoFLEX) using FlowJo VX software.

\section{TUNEL Staining}

TUNEL staining was performed using a situ one-step immunofluorescence TUNEL apoptosis assay kit (Beyotime Biotechnology, China) according to the manufacturer's instructions. Cy3-labeled TUNEL positive cells were observed under fluorescence microscope (Olympus IX710 Camera, Japan). Red fluorescent cells were defined as apoptotic cells. For quantification of apoptotic cells, three slices of the ischemic cerebral cortex in each animal were analyzed. The number of apoptosis cells in five fields of each slice at $200 \times$ magnification was measured using the Image Pro Plus software.

\section{Western Blot}

Brain samples ( $\mathrm{n}=4$ for each group) were separated from the cortex including both the penumbra and ischemic core regions, and protein was extracted with RIPA lysis buffer (Beyotime Biotechnology) containing 1\% protease inhibitors (sodium fluoride) and 1\% phosphatase inhibitors (sodium orthovanadate). Protein concentration was determined using a BCA protein assay kit. The protein from each sample was separated on $10 \%$ sodium dodecyl sulfate polyacrylamide gel 
electrophoresis gels, then transferred onto a PVDF membrane. The membrane was incubated in blocking buffer for $1 \mathrm{hr}$ at room temperature, and was then incubated overnight at $4^{\circ} \mathrm{C}$ with primary antibodies against NF- $\mathrm{kB}$ p-P65 (Cell Signaling), NF-kB P65 (Cell Signaling), p-ERK (Cell Signaling), ERK (Cell Signaling), NLRP1 (Cell Signaling), NLRP3 (Cell Signaling), caspase-1 (Abcam), IL-1 $\beta$ (Abcam), Cleaved caspase-3 (Cell Signaling), and $\beta$-actin (Cell Signaling). After washing three times ( 5 min per wash) with Tris-buffered saline, the membrane was incubated with secondary antibodies against the primary antibody for $1 \mathrm{hr}$ at room temperature. The membrane was washed with Trisbuffered saline and scanned using the gel imaging system (Protein Simple, FluorChem Q). Quantification of protein levels was achieved by densitometry analysis using Image Pro Plus 6.0 software. In detail, the densitometry of p-P65 $\mathrm{NF}-\kappa \mathrm{B}$ and $\mathrm{p}$-ERK (MAPK) signaling proteins divided by the densitometry of its corresponding "total protein" indicated the activation of NF- $\mathrm{KB}$ and ERK signaling. The densitometry of proteins caspase-1, mature IL-1 $\beta$, NLRP3 inflammasome, NLRP1 inflammasome and cleaved caspase-3 was determined by the densitometry of its corresponding $\beta$-actin, which was used as a loading control.

\section{Statistical Analysis}

Data analysis was performed using the GraphPad Prism 6 software. The data were represented as mean \pm SEM. Statistical analysis of the data was performed using oneway ANOVA followed by Student-Newman-Keuls post hoc comparisons. $P<0.05$ was considered significant.

\section{Results}

\section{LT Treatment Alleviates Neurological Deficits}

Neurological deficits were assessed by two observers in a blind manner at $48 \mathrm{hrs}$ after reperfusion. Conformity assessment certificated that there was no significant difference in behavior scores of the experimental animals at 2 hrs after the tMCAO operation. At $48 \mathrm{hrs}$ after reperfusion, the rats received tMCAO operation showed severe neurologic impairment, relative to rats in the Sham group $(P<0.05$, Figure 1A). LT treatment significantly reduced the neurology deficits score in tMCAO rats at $48 \mathrm{hrs}$ after reperfusion $(P<0.05$, Figure $1 \mathrm{~A})$.

The sensorimotor function of rats was assessed by tape removal test following neurology deficits score. As shown in Figure 1B, the tape removal latency time in the Sham group was $9.9 \pm 0.924 \mathrm{~s}$ at $48 \mathrm{hrs}$ after sham operation. The tape removal latency time increased significantly in the tMCAO $(139.3 \pm 9.996 \mathrm{~s})$ and $\mathrm{tMCAO}+\mathrm{LT}(103.7 \pm 11.87 \mathrm{~s})$ groups, respectively, at $48 \mathrm{hrs}$ after ischemia and reperfusion operation, compared with that in the Sham group $(P<0.05)$. LT treatment significantly decreased the latency time of tMCAO rats $(P<0.05)$.

\section{LT Treatment Reduces Infarct Volume}

Infarct volume was calculated using TTC-stained brain sections. Representative images of TTC staining of experimental rats are shown in Figure 2A. Compared with rats in the Sham group, rats in the $\mathrm{MCAO}$ group showed a significantly larger infarct volume at $48 \mathrm{hrs}$ after reperfusion $(P<0.05$, Figure 2B). The rats in the tMCAO+LT group exhibited a significant reduction of infarct volume, compared to rats in the tMCAO group $(P<0.05$, Figure $2 \mathrm{~B})$.

\section{LT Treatment Modulates the Differentiation of Tregs Subsets}

In order to evaluate the effects of LT treatment on the immune response of tMCAO rats, we separated monocytes from peripheral blood and analyzed the proportion of $\mathrm{CD} 4^{+} \mathrm{CD} 25^{+}$and $\mathrm{CD} 4{ }^{+} \mathrm{CD} 25^{+}$Foxp $3^{+}$Tregs by flow cytometry. As shown in Figure 3A and C, we observed that the percentage of $\mathrm{CD} 4{ }^{+} \mathrm{CD} 25^{+}$Tregs significantly increased at $48 \mathrm{hrs}$ after tMCAO $(P<0.05)$, and LT treatment did not change the percentage of $\mathrm{CD} 4^{+} \mathrm{CD} 25^{+}$Tregs in tMCAO rats. The proportion of $\mathrm{CD} 4^{+} \mathrm{CD} 25^{+} \mathrm{Foxp} 3^{+}$Tregs was markedly increased at $48 \mathrm{hrs}$ after tMCAO in the tMCAO group relative to that in the Sham group, and LT treatment further increased the percentage of $\mathrm{CD} 4^{+} \mathrm{CD} 25^{+} \mathrm{Foxp}^{+}$Tregs in tMCAO rats $(P<0.05$, Figure $3 \mathrm{~B}$ and $\mathrm{D})$.

\section{LT Treatment Alleviates the Inflammasome Activity}

In this study, we investigated the effect of LT treatment on immune-inflammatory activity, and the expression of several inflammasome proteins (caspase-1, mature IL-1 $\beta$, NLRP1 and NLRP3) was detected in brain tissues (cerebral cortex) following $48 \mathrm{hrs}$ of tMCAO. Western blot results demonstrated that protein levels of caspase-1, mature IL-1 $\beta$, NLRP1 and NLRP3 were significantly increased at $48 \mathrm{hrs}$ after tMCAO, compared with that in the Sham group $(P<0.05$, Figure 4A-D). LT treatment could remarkably reduce the protein levels of caspase-1, mature IL-1 $\beta$ and NLRP3 in tMCAO rats $(P<0.05$, Figure $4 \mathrm{~A}-\mathrm{C})$. LT treatment had no 

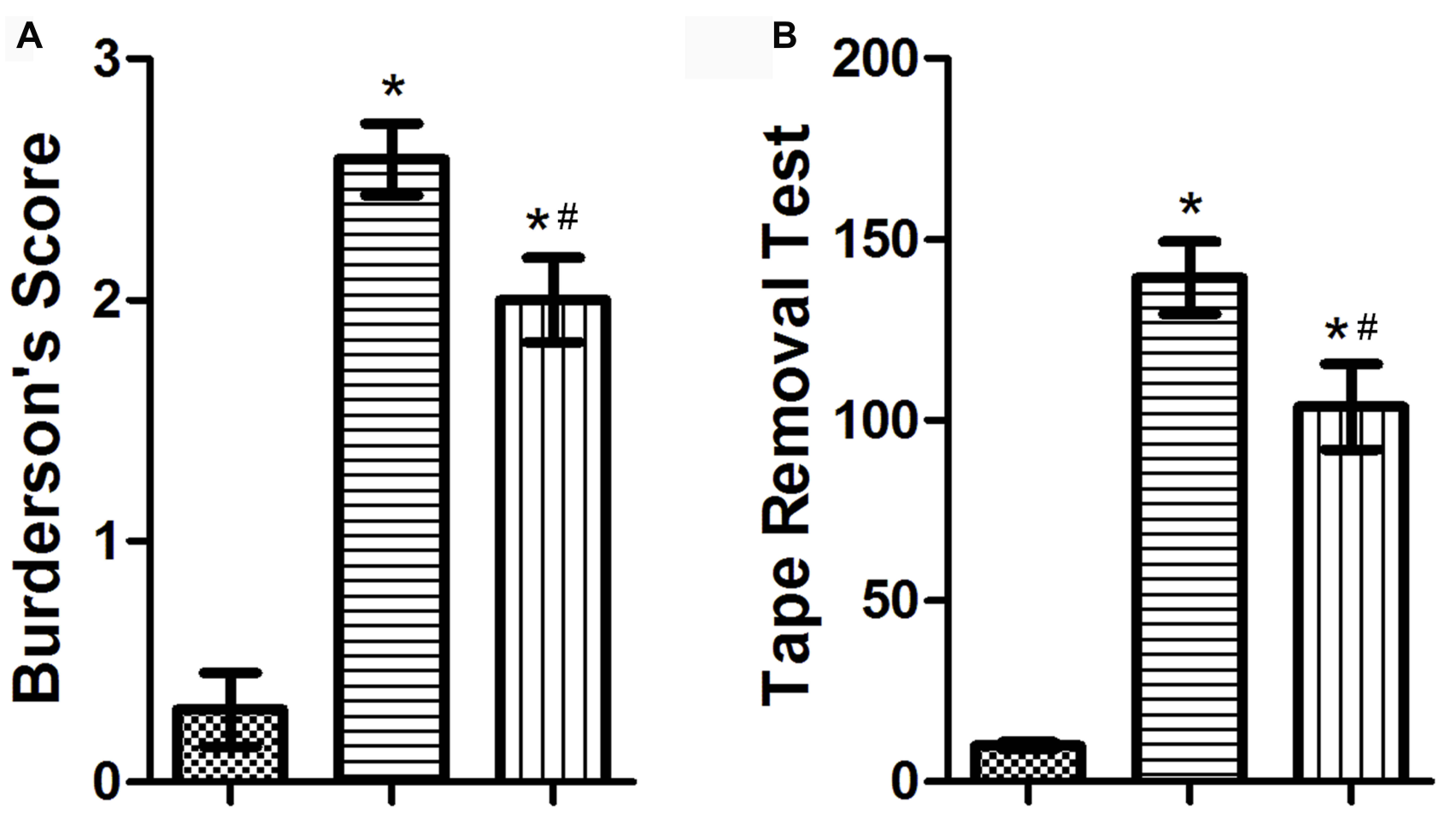

\section{Sham 罒}

Figure I Effect of the transplantation of lymphocytes co-cultured with HCB-SCs on neurological deficits at 48 hrs after stroke in rats. (A): Burderson's score. (B): Sensorimotor function test by tape removal. ${ }^{*} \mathrm{P}<0.05$ vs Sham group; ${ }^{\#} \mathrm{P}<0.05$ vs tMCAO group.

significant effect on the expression of NLRP1 in tMCAO rats $(P>0.05$, Figure 4D).

\section{LT Treatment Modulates the Immune Response by ERK and NF-kB Signalings}

We investigated the effect of LT treatment on the activation of intracellular NF- $\kappa \mathrm{B}$ and ERK signaling pathways. Our results demonstrated that the ratio of p-ERK/total ERK and

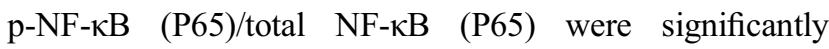
increased in the tMCAO group than that in the Sham group $(P<0.05$, Figure $4 \mathrm{E}$ and $\mathrm{F})$. LT treatment reduced the ratio of

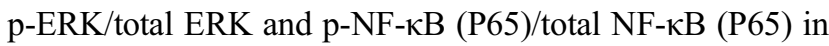
tMCAO rats $(P<0.05$, Figure $4 \mathrm{E}$ and $\mathrm{F})$. The results suggested that LT treatment could reduce the phosphorylation of NF- $\mathrm{BB}$ (P65) and ERK, and inhibit the activation of ERK and NF- $\kappa \mathrm{B}$ signaling pathways.

\section{LT Treatment Protects Neurons Against tMCAO-Induced Apoptosis}

To elucidate the anti-apoptotic effect of LT treatment, the expression level of cleaved caspase- 3 was determined by
Western blot. The result showed that the protein level of cleaved caspase- 3 in the tMCAO group was higher than that in the Sham group $(P<0.05$, Figure $4 \mathrm{G})$. LT treatment could significantly decrease the level of cleaved caspase-3 in tMCAO rats $(P<0.05$, Figure $4 \mathrm{G})$.

To further study the effect of LT treatment on neuron apoptosis, TUNEL staining was performed to analyze the number of apoptotic cells in ischemic cerebral cortex. The results showed that the number of apoptosis cells in cortex were markedly increased in mice with tMCAO compared with that with Sham operation $(P<0.05$, Figure 5). LT treatment significantly decreased the number of apoptosis cells in ischemic cerebral cortex $(P<0.05$, Figure 5), suggesting that the transplantation of lymphocytes co-cultured with HCB-SCs might protect neurons against tMCAOinduced apoptosis.

\section{Discussion}

Inflammatory processes are responsible for the development of cerebral ischemia and the magnification of brain damage after reperfusion. ${ }^{34}$ Modulating the immune inflammatory response after cerebral ischemia is deemed to be a novel 

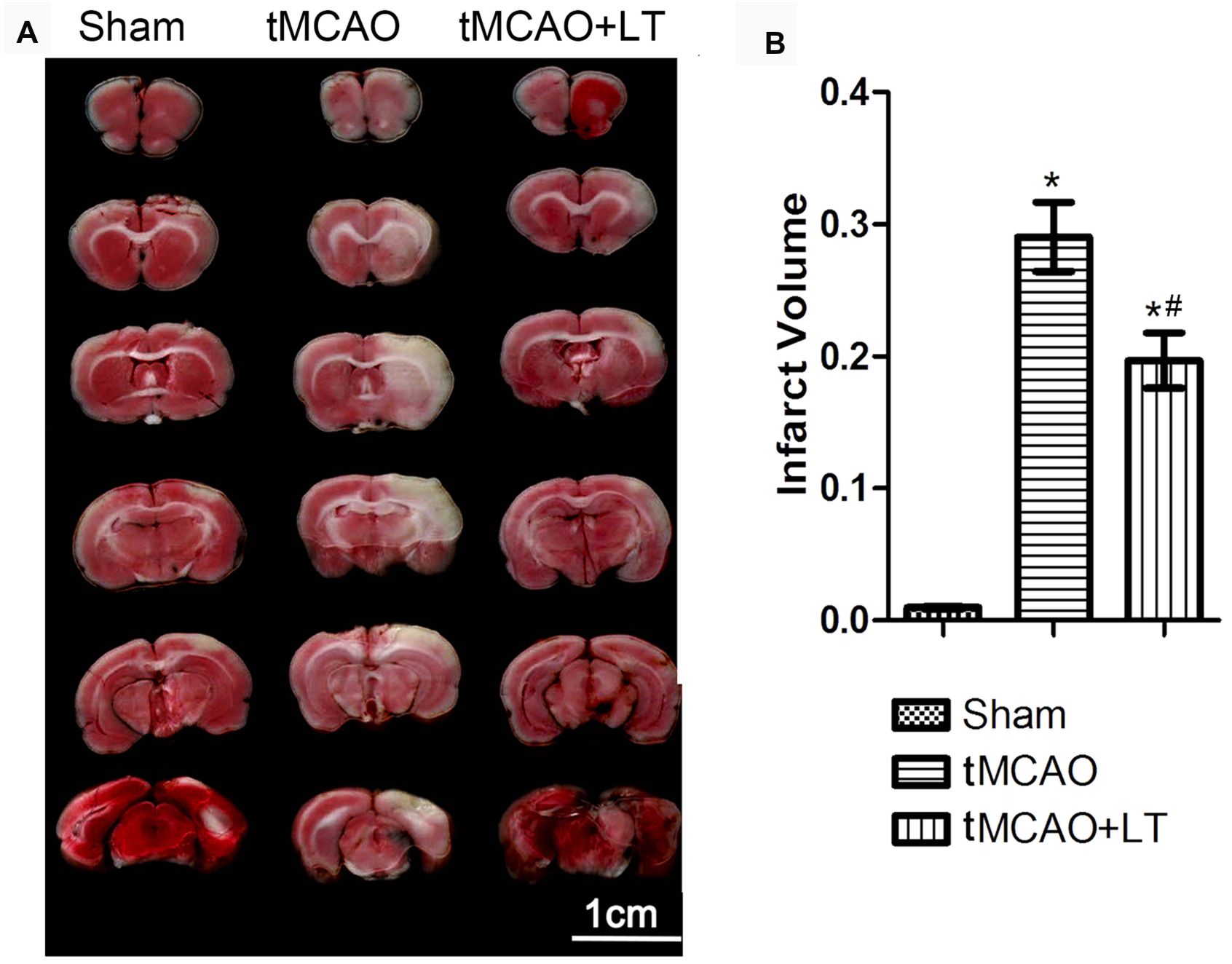

Q Sham ЕtMCAO 메 tMCAO+LT

Figure 2 Effect of the transplantation of lymphocytes co-cultured with HCB-SCs on infarct volume. (A): Representative images of TTC-stained brains at 48 hrs after stroke. (B): Quantification of brain infarct volume. ${ }^{* P}<0.05$ vs Sham group; ${ }^{*} P<0.05$ vs tMCAO group.

therapeutic strategy for ischemic stroke. Tregs have been characterized as inflammation-limiting protective cells in disease conditions, and play critical parts in maintaining post-stroke immune homeostasis and ameliorating inflammatory collateral damage. ${ }^{35}$ Our previous study provided evidence that lymphocytes co-cultured with HCB-SCs could modulate the number of Tregs, reduce pro-inflammatory cytokines, and improve anti-inflammatory cytokines in peripheral of APP/PS1 mice. ${ }^{25}$ In our study, lymphocytes cocultured with HCB-SCs exhibited the neuroprotective effects on ischemic stroke-induced inflammatory response and cerebral injury.

The transplantation of lymphocytes co-cultured with HCB-SCs could modulate peripheral inflammatory response by increasing the proportion of Foxp $3^{+}$Tregs in tMCAO rats. Ischemic infarct is accompanied by the activation of innate immune cascades, during which the blood-brain barrier is compromised and peripheral leukocytes infiltrate into the brain leading to substantial secondary damage. ${ }^{6}$ Tregs exert immune-modulating effects by either direct contact with the suppressed cells or release of immunosuppressive cytokines. Despite the large variability of Treg function and phenotype, the naturally occurring $\mathrm{CD} 4^{+} \mathrm{CD} 25^{+} \mathrm{Foxp}^{+}$Tregs are the best investigated population. ${ }^{36} \mathrm{CD} 4^{+} \mathrm{CD} 25^{+} \mathrm{Foxp}^{+}{ }^{+}$Tregs play a key part in controlling immune responses in various systemic and central nervous system inflammatory diseases. ${ }^{36}$ It has been proved that Tregs are major cerebroprotective immunomodulators of post-ischemic inflammatory brain damage. ${ }^{14}$ Therefore, increasing the proportion of $\mathrm{CD}^{+}{ }^{+} \mathrm{CD} 25^{+} \mathrm{Foxp}^{+}$Tregs might be an effective immunomodulatory therapy for stroke.

Our study indicated that the transplantation of lymphocytes co-cultured with HCB-SCs could reduce stroke-induced brain injury. HCB-SCs are a novel and phenotypically distinct 

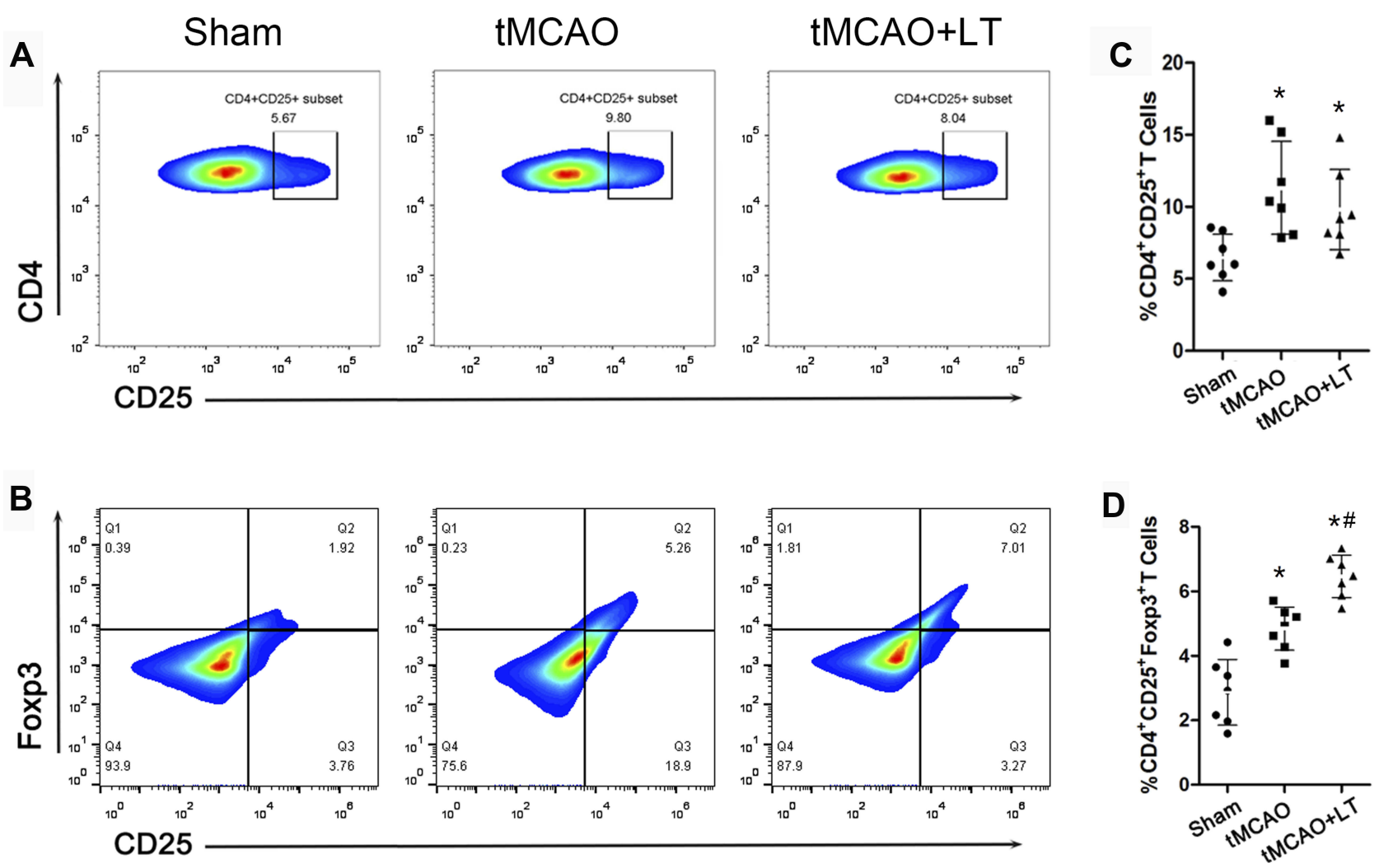

Figure 3 Effect of the transplantation of lymphocytes co-cultured with HCB-SCs on modulating the differentiation of Tregs. Representative flow cytometry plots gated on $\mathrm{CD} 4^{+} \mathrm{CD} 25^{+} \mathrm{T}$ cells $(\mathbf{A})$ and $\mathrm{CD} 4^{+} \mathrm{CD} 25^{+} \mathrm{Foxp} 3^{+} \mathrm{T}$ cells $(\mathbf{B})$. Percentages of CD4 ${ }^{+} \mathrm{CD} 25^{+} \mathrm{T}$ cells $(\mathbf{C})$ and CD $4^{+} \mathrm{CD} 25^{+} \mathrm{Foxp} 3^{+} \mathrm{T}$ cells $(\mathbf{D}) .{ }^{*} P<0.05$ vs Sham group; ${ }^{\#} \mathrm{P}<0.05$ vs tMCAO group.

subset of stem cells that are identified from the umbilical cord blood. ${ }^{26}$ A growing body of evidence has demonstrated the immune therapeutic potential of HCB-SCs by regulating T lymphocytes. ${ }^{29,30,37}$ HCB-SCs may restore immune balance by altering cytokines (such as IL-10 and TGF- $\beta$ ) or cell composition (such as Tregs). The immune modulation of HCB-SCs to lymphocytes has also successfully detected in the therapeutic potential of Type 1 diabetes. ${ }^{37,38} \mathrm{He}$ et $\mathrm{al}^{25}$ discovered that HCB-SCs could increase the proportion of Tregs in peripheral lymphocytes and could enhance the antiinflammatory function in vitro. These results imply the immune-modulating potential of HCB-SCs by co-culturing with lymphocytes for stroke.

In this study, the transplantation of lymphocytes cocultured with HCB-SCs attenuated ischemic stroke-induced NLRP3 inflammasomes and the corresponding downstream pro-inflammatory cytokines, such as caspase- 1 and IL-1 $\beta$, and pro-apoptotic protein cleaved caspase-3. Inflammasomes are multi-protein complexes that are activated as part of the innate immune response, and can trigger the maturation of caspase- 1 followed by the production of IL- $1 \beta .^{39,40}$ The NLRP inflammasomes are cytosolic macromolecular complexes composed of the NLRP receptor, ASC (apoptosis-associated speck-like protein containing a caspase recruitment domain), and precursor caspase-1. ${ }^{39}$ It has been demonstrated that ischemia-like conditions can increase the level of NLRP inflammasomes and IL-1 $\beta{ }^{41}$ Fann et $\mathrm{al}^{41}$ showed that the NLRP1 and NLRP3 inflammasomes play a major role in neuronal cell death and sensorimotor impairment following stroke. IL-1 $\beta$ has also been strongly implicated in the pathogenesis of ischemic brain as a neurotoxic mediator. ${ }^{6,39}$ Therefore, we attempted to determine whether the levels of IL-1 $\beta$, caspase-1, NLRP1 and NLRP3 in the lesion side of cerebral hemispheres were changed by the transplantation of lymphocytes co-cultured with HCB-SCs. This study showed that the transplantation of co-cultured lymphocytes reduced the expression of NLRP3 rather than NLRP1, suggesting that NLRP3 might involve in the modulation of inflammatory response under the transplantation treatment.

Our study found that the transplantation of lymphocytes co-cultured with HCB-SCs decreased the expression of pre-IL $-1 \beta$ (data not shown) and mature IL-1 $\beta$, indicating that the transplantation promoted the mature process of IL- $1 \beta$ by not only NLRP3 and caspase-1 inflammasome signaling pathway, 
A
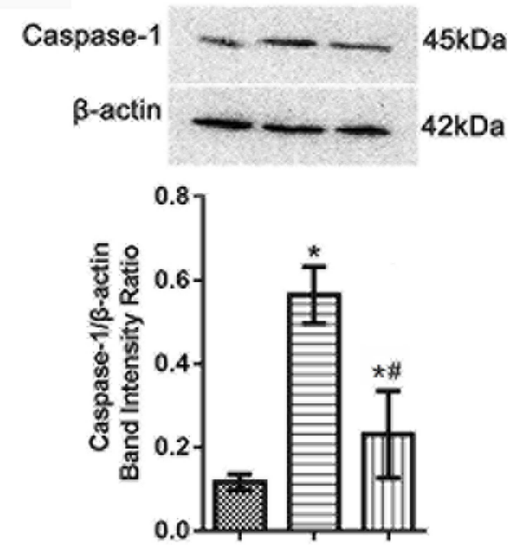

D
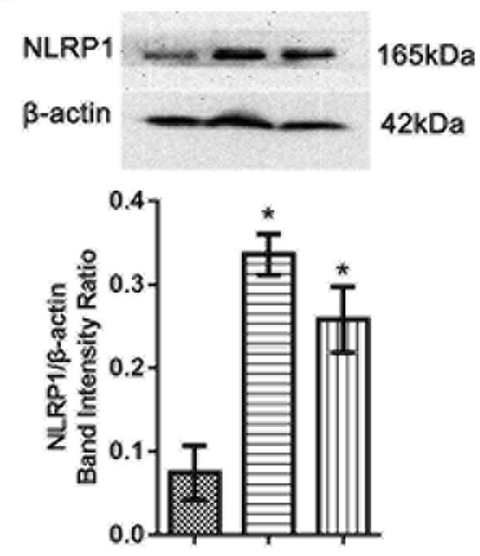

B
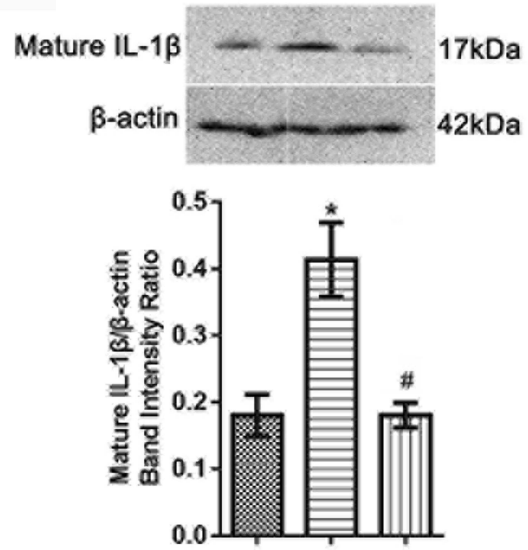

$\mathbf{E}$
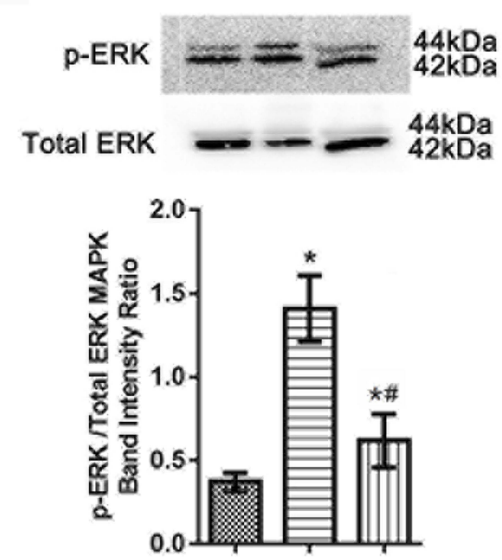

C
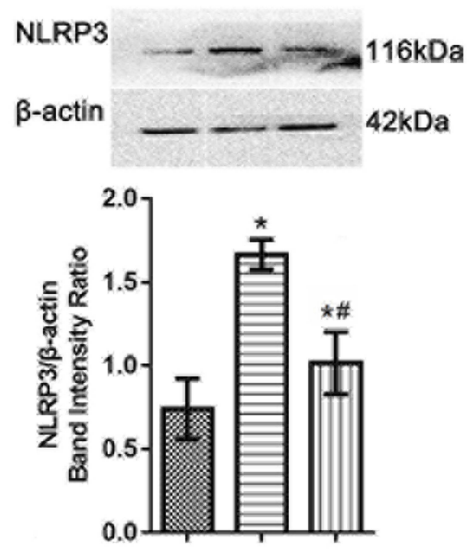

$\mathbf{F}$
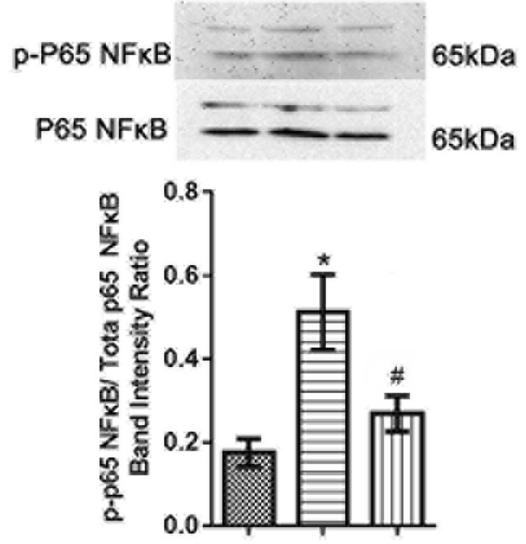

G
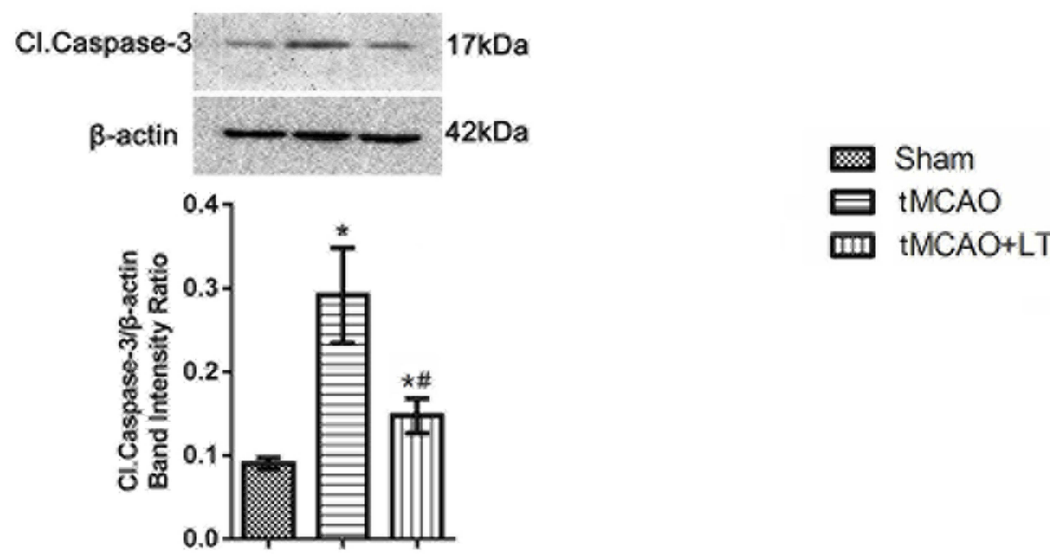

Figure 4 Effect of the transplantation of lymphocytes co-cultured with HCB-SCs on the inflammatory activity. Western blot shows the protein levels of caspase-I (A), mature IL-I $\boldsymbol{\beta}$ (B), NLRP3 (C), NLRPI (D), p-ERK/ERK (E), NF-KB p-P65/P65 (F), and cleaved caspase-3 (G). The NF-kB specific band is located at 65 kDa, and a nonspecific band can be seen at a molecular weight of $72 \mathrm{kDa}(\mathbf{F})$. ${ }^{* P}<0.05$ vs Sham group; ${ }^{\#} P<0.05$ vs tMCAO group.

but also more extensive inflammatory signaling pathways. Therefore, we further tested NF- $\mathrm{kB}$ and ERK (MAPK) signaling molecules. Accumulating evidence has indicated that the activation of IL-1 receptor 1 leads to the activation of intracellular NF- $\mathrm{KB}$ and ERK signaling pathways through a process known as "priming". ${ }^{41-43}$ Meanwhile, an increase in expression of NLRP1 and NLRP3 inflammasomes in brain cells under ischemic conditions may also be induced by the 

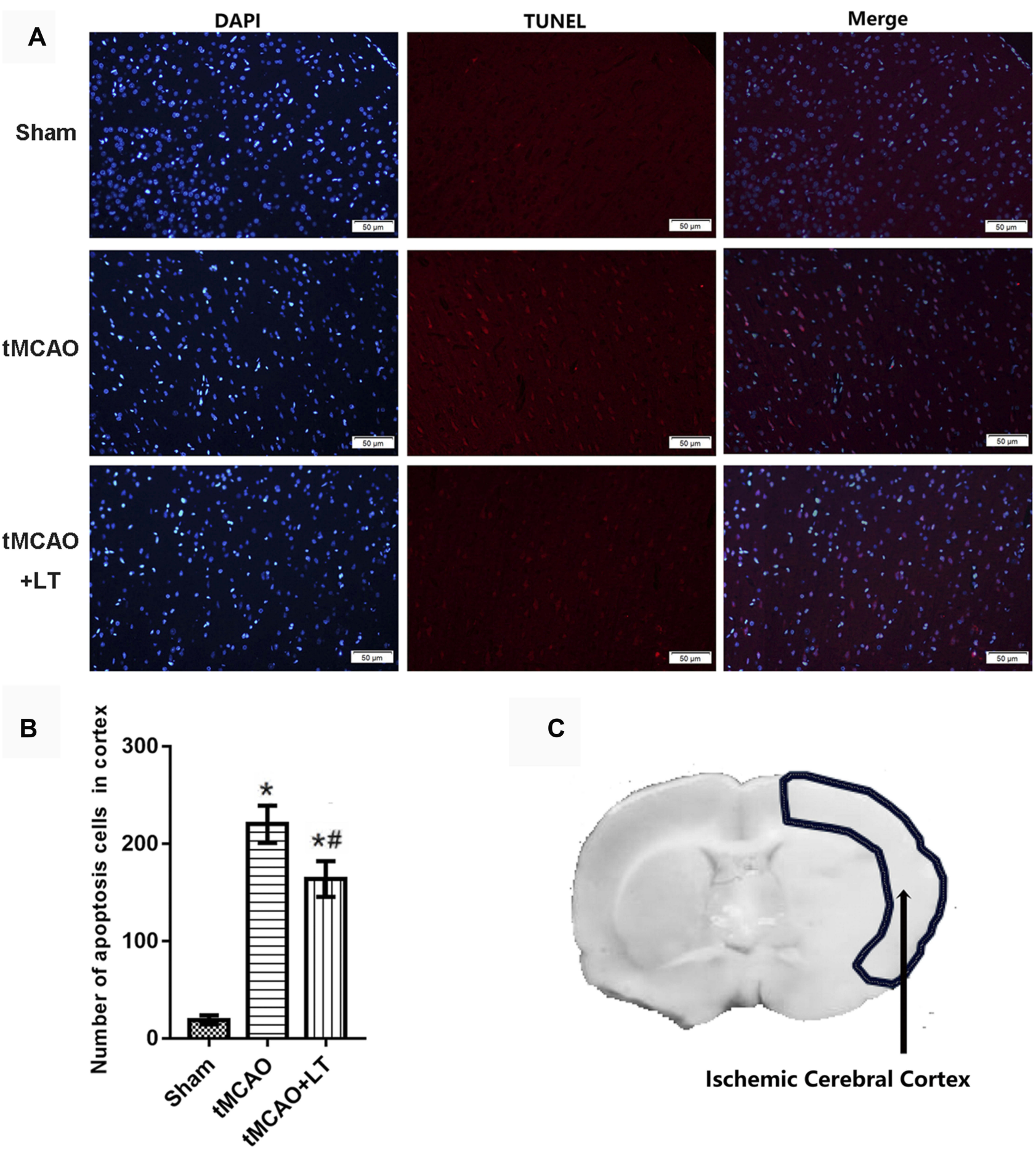

Ischemic Cerebral Cortex

Figure 5 Effect of the transplantation of lymphocytes co-cultured with HCB-SCs on cell apoptosis in the ischemic cerebral cortex. TUNEL staining is used to analyze number of apoptosis cells in cerebral cortex. Representative images of TUNEL staining (A). The statistical graph of the number of apoptosis cells (B). Schematic of the brain shows the detection region (C). ${ }^{* P}<0.05$ vs Sham group; ${ }^{\#} P<0.05$ vs tMCAO group.

activation of NF- $\mathrm{BB}$ and MAPK signaling pathways. ${ }^{42}$ Our current data showed that $\mathrm{TMCAO}$-induced activation of NF- $\mathrm{KB}$ and ERK signaling pathways could be significantly downregulated by the transplantation of lymphocytes co-cultured with HCB-SCs. Consequently, the activation of NF- $\mathrm{kB}$ and
ERK signaling pathways may further induce the protein expressions of NLRP3, and caspase-1 under ischemic conditions.

Furthermore, the activation of inflammasome pathway is proven to promote caspase-3-induced cell apoptosis. ${ }^{44}$ 
Thus, in addition to the anti-inflammation effects, we further examined the anti-apoptosis effect of the transplantation treatment. Caspase- 3 has been identified as a key mediator of apoptosis in animal models of ischemic stroke. $^{45}$ Our data showed that the transplantation treatment significantly attenuated ischemia-induced activation of caspase- 3 at 48 hrs after tMCAO, and LT treatment decrease the level of cleaved caspase- 3 in tMCAO rats. TUNEL staining confirmed the effect of LT treatment on neuron apoptosis, suggesting that the transplantation of lymphocytes co-cultured with HCB-SCs might protect neurons by defeating tMCAO-induced cell apoptosis.

\section{Conclusion}

Our study provided evidence that the transplantation of lymphocytes co-cultured with HCB-SCs could elevate the proportion of $\mathrm{CD} 4^{+} \mathrm{CD} 25^{+} \mathrm{Foxp} 3^{+}$Tregs in peripheral blood and attenuate inflammatory reaction after tMCAO. Notably, the neuroprotective effect of the lymphocytes cocultured with HCB-SCs was closely related to the decrease of NLRP3 inflammasome and associated inflammatory factors. Future experimental and preclinical researches may identify the transplantation lymphocytes co-cultured with HCB-SCs as a novel therapeutic strategy for neurovascular diseases.

\section{Acknowledgments}

We thank Yi He, Guitao Zhang, Nana Huang, and Mingming Guo for data curation and methodology assistance. We appreciate the central laboratory of the Jinan Central Hospital and School of Medicine of Shandong University for providing experimental places and facilities.

\section{Author Contributions}

All authors contributed to data analysis, drafting or revising the article, gave final approval of the version to be published, and agree to be accountable for all aspects of the work. Yanxin Zhao and Tianrui Zhu should be considered as co-first authors.

\section{Funding}

This work was supported by Primary Research \& Development Plan of Shandong Province (Grant No. 2016GSF121044) and Nation Natural Science Foundation of China (Grant No. 81373635).

\section{Disclosure}

The authors report no conflicts of interest in this work.

\section{References}

1. Collaborators GBDS. Global, regional, and national burden of stroke, 1990-2016: a systematic analysis for the Global Burden of Disease Study 2016. Lancet Neurol. 2019;18(5):439-458. doi:10.1016/S14744422(19)30034-1

2. Rajsic S, Gothe H, Borba HH, et al. Economic burden of stroke: a systematic review on post-stroke care. Eur J Health Econ. 2019;20 (1):107-134. doi:10.1007/s10198-018-0984-0

3. Ovbiagele B, Goldstein LB, Higashida RT, et al. Forecasting the future of stroke in the United States: a policy statement from the American Heart Association and American Stroke Association. Stroke. 2013;44(8):2361-2375. doi:10.1161/STR.0b013e31829734f2

4. Hankey GJ. Stroke. Lancet. 2017;389(10069):641-654. doi:10.1016/ S0140-6736(16)30962-X

5. Wardlaw JM, Bath PM. Stroke research in 2018: extended time windows, refined benefit, and lifestyle prevention targets. Lancet Neurol. 2019;18(1):2-3. doi:10.1016/S1474-4422(18)30457-5

6. Lambertsen KL, Finsen B, Clausen BH. Post-stroke inflammation-target or tool for therapy? Acta Neuropathol. 2019;137(5):693-714. doi:10.1007/s00401-018-1930-z

7. Zierath D, Schulze J, Kunze A, et al. The immunologic profile of adoptively transferred lymphocytes influences stroke outcome of recipients. J Neuroimmunol. 2013;263(1-2):28-34. doi:10.1016/j. jneuroim.2013.07.014

8. Offner H, Hurn PD. A novel hypothesis: regulatory B lymphocytes shape outcome from experimental stroke. Trans Stroke Res. 2012;3 (3):324-330. doi:10.1007/s12975-012-0187-4

9. Ducruet AF, Connolly ES Jr. Targeting lymphocytes in ischemic stroke. World Neurosur. 2011;76(3-4):212-214. doi:10.1016/j.wneu.2011. 06.060

10. Liesz A, Zhou W, Mracsko E, et al. Inhibition of lymphocyte trafficking shields the brain against deleterious neuroinflammation after stroke. Brain. 2011;134(Pt 3):704-720. doi:10.1093/brain/awr008

11. Shichita T, Sugiyama Y, Ooboshi H, et al. Pivotal role of cerebral interleukin-17-producing gammadeltaT cells in the delayed phase of ischemic brain injury. Nat Med. 2009;15(8):946-950. doi:10.1038/ nm.1999

12. Liesz A, Hu X, Kleinschnitz C, Offner H. Functional role of regulatory lymphocytes in stroke: facts and controversies. Stroke. 2015;46 (5):1422-1430. doi:10.1161/STROKEAHA.114.008608

13. Ren X, Akiyoshi K, Dziennis S, et al. Regulatory B cells limit CNS inflammation and neurologic deficits in murine experimental stroke. $J$ Neurosci. 2011;31(23):8556-8563. doi:10.1523/JNEUROSCI.162311.2011

14. Liesz A, Suri-Payer E, Veltkamp C, et al. Regulatory T cells are key cerebroprotective immunomodulators in acute experimental stroke. Nat Med. 2009;15(2):192-199. doi:10.1038/nm.1927

15. Shevach EM. Mechanisms of foxp3 $+\mathrm{T}$ regulatory cell-mediated suppression. Immunity. 2009;30(5):636-645. doi:10.1016/j. immuni.2009.04.010

16. McGeachy MJ, Stephens LA, Anderton SM. Natural recovery and protection from autoimmune encephalomyelitis: contribution of CD4+CD25+ regulatory cells within the central nervous system. J Immunol. 2005;175 (5):3025-3032. doi:10.4049/jimmunol.175.5.3025

17. Shinozuka K, Dailey T, Tajiri N, Ishikawa H, Kaneko Y, Borlongan CV. Stem cell transplantation for neuroprotection in stroke. Brain Sci. 2013;3(1):239-261. doi:10.3390/brainsci3010239

18. Kozlowska H, Jablonka J, Janowski M, Jurga M, Kossut M, DomanskaJanik K. Transplantation of a novel human cord blood-derived neural-like stem cell line in a rat model of cortical infarct. Stem Cells Dev. 2007;16 (3):481-488. doi:10.1089/scd.2007.9993

19. Liu K, Guo L, Zhou Z, Pan M, Yan C. Mesenchymal stem cells transfer mitochondria into cerebral microvasculature and promote recovery from ischemic stroke. Microvasc Res. 2019;123:74-80. doi:10.1016/j.mvr.2019.01.001 
20. Chang YC, Shyu WC, Lin SZ, Li H. Regenerative therapy for stroke. Cell Trans. 2007;16(2):171-181. doi:10.3727/000000007783464669

21. Haas S, Weidner N, Winkler J. Adult stem cell therapy in stroke. Curr Opin Neurol. 2005;18(1):59-64. doi:10.1097/00019052-20050200000012

22. Sanberg PR, Willing AE, Garbuzova-Davis S, et al. Umbilical cord blood-derived stem cells and brain repair. Ann $N$ Y Acad Sci. 2005;1049:67-83. doi:10.1196/annals.1334.008

23. Koh SH, Kim KS, Choi MR, et al. Implantation of human umbilical cord-derived mesenchymal stem cells as a neuroprotective therapy for ischemic stroke in rats. Brain Res. 2008;1229:233-248. doi:10.1016/j.brainres.2008.06.087

24. Kim SW, Han H, Chae GT, et al. Successful stem cell therapy using umbilical cord blood-derived multipotent stem cells for Buerger's disease and ischemic limb disease animal model. Stem Cells. 2006;24(6):1620-1626. doi:10.1634/stemcells.2005-0365

25. He Y, Li H, Zhang F, et al. Immunotherapeutic effects of lymphocytes co-cultured with human cord blood-derived multipotent stem cells transplantation on APP/PS1 mice. Behav Brain Res. 2016;315:94-102. doi:10.1016/j.bbr.2016.08.025

26. Zhao Y, Wang H, Mazzone T. Identification of stem cells from human umbilical cord blood with embryonic and hematopoietic characteristics. Exp Cell Res. 2006;312(13):2454-2464. doi:10.1016/j.yexcr.2006.04.008

27. Vendrame M, Cassady J, Newcomb J, et al. Infusion of human umbilical cord blood cells in a rat model of stroke dose-dependently rescues behavioral deficits and reduces infarct volume. Stroke. 2004;35 (10):2390-2395. doi:10.1161/01.STR.0000141681.06735.9b

28. Lewis ID. Clinical and experimental uses of umbilical cord blood. Int Med J. 2002;32(12):601-609. doi:10.1046/j.1445-5994.2002.00276.x

29. Erices AA, Allers CI, Conget PA, Rojas CV, Minguell JJ. Human cord blood-derived mesenchymal stem cells home and survive in the marrow of immunodeficient mice after systemic infusion. Cell Trans. 2003;12(6):555-561. doi:10.3727/000000003108747154

30. Park DH, Borlongan CV, Willing AE, et al. Human umbilical cord blood cell grafts for brain ischemia. Cell Trans. 2009;18(9):985-998. doi: $10.3727 / 096368909 X 471279$

31. Traggiai E, Chicha L, Mazzucchelli L, et al. Development of a human adaptive immune system in cord blood cell-transplanted mice. Science. 2004;304(5667):104-107. doi:10.1126/science.1093933

32. Bederson JB, Pitts LH, Tsuji M, Nishimura MC, Davis RL, Bartkowski H. Rat middle cerebral artery occlusion: evaluation of the model and development of a neurologic examination. Stroke. 1986;17(3):472-476. doi:10.1161/01.STR.17.3.472

33. Bouet V, Freret T, Toutain J, Divoux D, Boulouard M, SchumannBard P. Sensorimotor and cognitive deficits after transient middle cerebral artery occlusion in the mouse. Exp Neurol. 2007;203 (2):555-567. doi:10.1016/j.expneurol.2006.09.006
34. Zhao J, Mou Y, Bernstock JD, et al. Synthetic oligodeoxynucleotides containing multiple telemeric ttaggg motifs suppress inflammasome activity in macrophages subjected to oxygen and glucose deprivation and reduce ischemic brain injury in stroke-prone spontaneously hypertensive rats. PLoS One. 2015;10(10):e0140772. doi:10.1371/ journal.pone.0140772

35. Liesz A, Kleinschnitz C. Regulatory $\mathrm{T}$ cells in post-stroke immune homeostasis. Trans Stroke Res. 2016;7(4):313-321. doi:10.1007/ s12975-016-0465-7

36. Shevach EM, DiPaolo RA, Andersson J, Zhao DM, Stephens GL, Thornton AM. The lifestyle of naturally occurring CD4+ CD25+ Foxp3 + regulatory T cells. Immunol Rev. 2006;212:60-73. doi:10.1111/j.01052896.2006.00415.x

37. Zhao Y, Jiang Z, Zhao T, et al. Reversal of type 1 diabetes via islet beta cell regeneration following immune modulation by cord blood-derived multipotent stem cells. BMC Med. 2012;10:3. doi:10.1186/1741-7015-10-3

38. He B, Li X, Yu H, Zhou Z. Therapeutic potential of umbilical cord blood cells for type 1 diabetes mellitus. J Diabetes. 2015;7(6):762-773. doi:10.1111/1753-0407.12286

39. Walsh JG, Muruve DA, Power C. Inflammasomes in the CNS. Nat Rev Neuroscience. 2014;15(2):84-97. doi:10.1038/nrn3638

40. Mamik MK, Power C. Inflammasomes in neurological diseases: emerging pathogenic and therapeutic concepts. Brain. 2017;140 (9):2273-2285. doi:10.1093/brain/awx133

41. Fann DY, Lee SY, Manzanero S, et al. Intravenous immunoglobulin suppresses NLRP1 and NLRP3 inflammasome-mediated neuronal death in ischemic stroke. Cell Death Dis. 2013;4:e790. doi:10.1038/ cddis.2013.326

42. Fann DY, Santro T, Manzanero S, et al. Intermittent fasting attenuates inflammasome activity in ischemic stroke. Exp Neurol. 2014;257:114-119. doi:10.1016/j.expneurol.2014.04.017

43. Subramaniam S, Unsicker K. ERK and cell death: ERK1/2 in neuronal death. FEBS J. 2010;277(1):22-29. doi:10.1111/j.1742-4658.2009. 07367.x

44. Sagulenko V, Vitak N, Vajjhala PR, Vince JE, Stacey KJ. Caspase-1 is an apical caspase leading to caspase-3 cleavage in the AIM2 inflammasome response, independent of caspase-8. J Mol Biol. 2018;430(2):238-247. doi:10.1016/j.jmb.2017.10.028

45. Broughton BR, Reutens DC, Sobey CG. Apoptotic mechanisms after cerebral ischemia. Stroke. 2009;40(5):e331-339. doi:10.1161/ STROKEAHA.108.531632
Clinical Interventions in Aging

\section{Publish your work in this journal}

Clinical Interventions in Aging is an international, peer-reviewed journal focusing on evidence-based reports on the value or lack thereof of treatments intended to prevent or delay the onset of maladaptive correlates of aging in human beings. This journal is indexed on PubMed Central, MedLine, CAS, Scopus and the Elsevier
Bibliographic databases. The manuscript management system is completely online and includes a very quick and fair peer-review system, which is all easy to use. Visit http://www.dovepress.com/ testimonials.php to read real quotes from published authors. 